\title{
Power Doppler Myocardial Contrast Echocardiography Using an Improved Multiple Frame Triggered Harmonic Angio Technique
}

Thippeswamy H. Murthy, M.D., Elizabeth Locricchio, B.S., R.D.C.S., Bettina Kuersten, M.D., Peng Li, M.D., Cheryl Baisch, B.S., and Mani A. Vannan, M.B.B.S.

The Division of Cardiology, Department of Internal Medicine, University of Michigan, Ann Arbor, Michigan

Although B-mode harmonic, intermittent-triggered myocardial contrast echocardiography (MCE) is a well-established technique, a variety of MCE techniques have been introduced recently to improve myocardial opacification. One such technique uses a power Doppler method in conjunction with multiple frame triggering (MFT), but has been limited by nonuniform microbubble destruction and blooming as well as motion artifacts. Utilizing two different contrast agents, Definity and Optison, we tested the feasibility of an improved version of Harmonic Angio MFT that utilizes a lower transmit frequency, reduced packet size, and more stringent wall filter in normal volunteers and in patients with known perfusion defects. The results showed that Harmonic Angio MFT produced fill frames with readily visible opacification and destruction frames with little visible opacification. The patterns of opacification also correlated with the expected perfusion patterns in both groups of subjects. Thus, Harmonic Angio MFT appears to be a promising new MCE technique. (ECHOCARDIOGRAPHY, Volume 18, April 2001)

echocardiography, contrast, Doppler, myocardial perfusion

B-mode harmonic, intermittent-triggered myocardial contrast echocardiography (MCE) is an established MCE technique; however it is hampered by a relatively low microbubble signal-to-tissue noise ratio. This is partly due to the harmonic response of tissue, which decreases the signal-to-noise ratio. The technique involves subtracting baseline noncontrast images from contrast images, which requires postprocessing of data, including frame-byframe image realignment. A variety of techniques were introduced recently to solve these problems, including Doppler-type techniques, ${ }^{1,2}$ real-time $\mathrm{MCE},{ }^{3,4}$ and algorithms, which capitalize on the nonlinear nature of the microbubble's harmonic response.

Harmonic Angio (Agilent Technologies, Andover, MA, USA) is a promising new technique that measures the power and not the velocity of the Doppler shift from a packet of multiple transmitted pulses with each scan line. Each

Supported by Agilent Technologies: Research Grant.

Address for correspondence and reprint requests: Mani Vannan, M.B.B.S., Womens L3119, University of Michigan Hospital, 1500 E. Medical Center Drive, Ann Arbor, MI 48109. Fax: 734-763-7390; E-mail: mvannan@umich.edu consecutive pulse leads to microbubble disruption, producing high velocity Doppler shifts. The lower velocity Doppler shifts of the tissue can be removed with a wall filter, thus increasing the microbubble signal-to-tissue noise ratio and possibly precluding the need to subtract baseline images.

However, Harmonic Angio is subject to artifacts from cardiac translation motion and blooming from excessive contrast administration. One solution to this problem is to use multiple frame triggering (MFT) in which two images are generated by transmission of two ultrasound impulses in rapid succession, the shortest interval between two frames being determined by the continuous imaging frame rate. The first frame of this dual frame technique simultaneously images and disrupts microbubbles and visually produces what has been referred to as the "fill frame." The second impulse disrupts the residual microbubbles and visually produces what has been referred to as the "destruction frame." The destruction frame is taken so soon after the fill frame that microbubbles do not have enough time to adequately replenish the myocardium. Thus, assuming complete microbubble destruction with the fill frame, the difference between the fill 
and destruction frames represents the myocardial microcirculatory signal. However, high mechanical index (MI) techniques, such as Harmonic Angio MFT (Agilent Technologies), produce heterogeneous microbubble destruction, resulting in greater signal intensity from the myocardial segments closer to the transducer due to nonuniform power in the field and attenuation from contrast in the left ventricular cavity. ${ }^{5}$ Furthermore, myocardial motion between the two frames produces artifacts.

We tested an improved version of Harmonic Angio MFT using a transducer with a lower transmit frequency, reduced packet size (5 pulses instead of 8), and a more stringent wall filter. A lower transmit frequency would be expected to produce fewer artifacts by providing better penetration and more uniform power in the field. A reduced packet size would be expected to result in less motion artifact due to better temporal resolution. The purpose of the study was to test whether this improved, low frequency Harmonic Angio technique could produce fill frames with readily visible myocardial opacification and destruction frames with uniform and complete microbubble destruction.

\section{Methods}

\section{Subject Population}

Harmonic Angio MFT was used to study 38 subjects with good apical acoustic windows. Twenty-eight healthy volunteers (21-33 years old, 12 female; 12 DMP-115 [Definity, Dupont Pharmaceuticals, North Billerica, MA, USA]; 16 Optison [Optison, Mallinkrodt, St. Louis, MO, USA]) were recruited by posted advertisements. Ten patients (43-81 years old, 4 female; 10 Optison) with a documented history of myocardial infarction by electrocardiogram (ECG) and creatine kinase (CPK) criteria were recruited from the inpatient cardiology service of the University of Michigan Hospital. All of the myocardial infarction patients underwent resting single photon emission computer tomography (SPECT) imaging. All subjects underwent an informed written consent process for the protocol that was approved by the institutional review board.

\section{MCE Technique}

The contrast agents used in this study were either DMP-115, given as a continuous infusion through a peripheral IV $(1300 \mu \mathrm{L}$ in $25 \mathrm{ml}$ of $0.9 \mathrm{NS}$ at $4-6 \mathrm{ml} / \mathrm{min})$, or Optison $(0.33$ $\mathrm{ml} / \mathrm{min}$ ). A commercially available ultrasound platform in conjunction with a prototype broadband transducer (S3) was used in this study. All subjects underwent resting MCE with high MI (1.1-1.3), triggered (1:4) Harmonic Angio MFT. The transmit focus was positioned in either the upper one-third or lower two-thirds of the imaging field. The two-dimensional gains were set to minimize the tissue signal. The color gain was set at the maximum value that resulted in minimal power Harmonic Angio signal in the baseline images. Once optimized, these settings were held constant throughout the study. During MFT, the first triggered frame was used as the fill frame and the next triggered frame was used as the destruction frame. The time interval between the fill and destruction frames was determined by the continuous imaging frame rate, which varied from $20-30 \mathrm{~Hz}$. All studies were recorded onto sVHS videotape as well digitally stored. Only loops obtained after a visible steady-state level of myocardial opacification were examined. The most representative loops from each study were used for examination.

\section{Results}

MCE was successfully performed throughout the complete duration of contrast infusion in all subjects without any adverse reactions. Readily discernible myocardial opacification was seen in all subjects. Furthermore, the contrast effect was noted throughout the duration of the infusion and lasted up to 2 minutes after the termination of the contrast infusion in several subjects.

Harmonic Angio MFT with Optison in healthy volunteers resulted in readily visible opacification in the fill frames and markedly reduced opacification in the destruction frames, as shown for three representative volunteers (Fig. 1). In each panel, the fill frame is on the left and the destruction frame is on the right. The fill frames show homogenous opacification with contrast while the destruction frames show a relatively uniform absence of contrast signal. Figure 2 shows two consecutive destruction frames in the same volunteer. In Figure $2 \mathrm{~A}$, the first of the two consecutive destruction frames was imaged $50 \mathrm{msec}$ after the fill frame is shown. Figure 2B shows the second of the two consecutive destruction frames which was imaged $50 \mathrm{msec}$ after the first destruction frame (i.e., $100 \mathrm{msec}$ after the fill frame). Both destruction frames visually appeared equal.

Harmonic Angio MFT with Definity in 
A
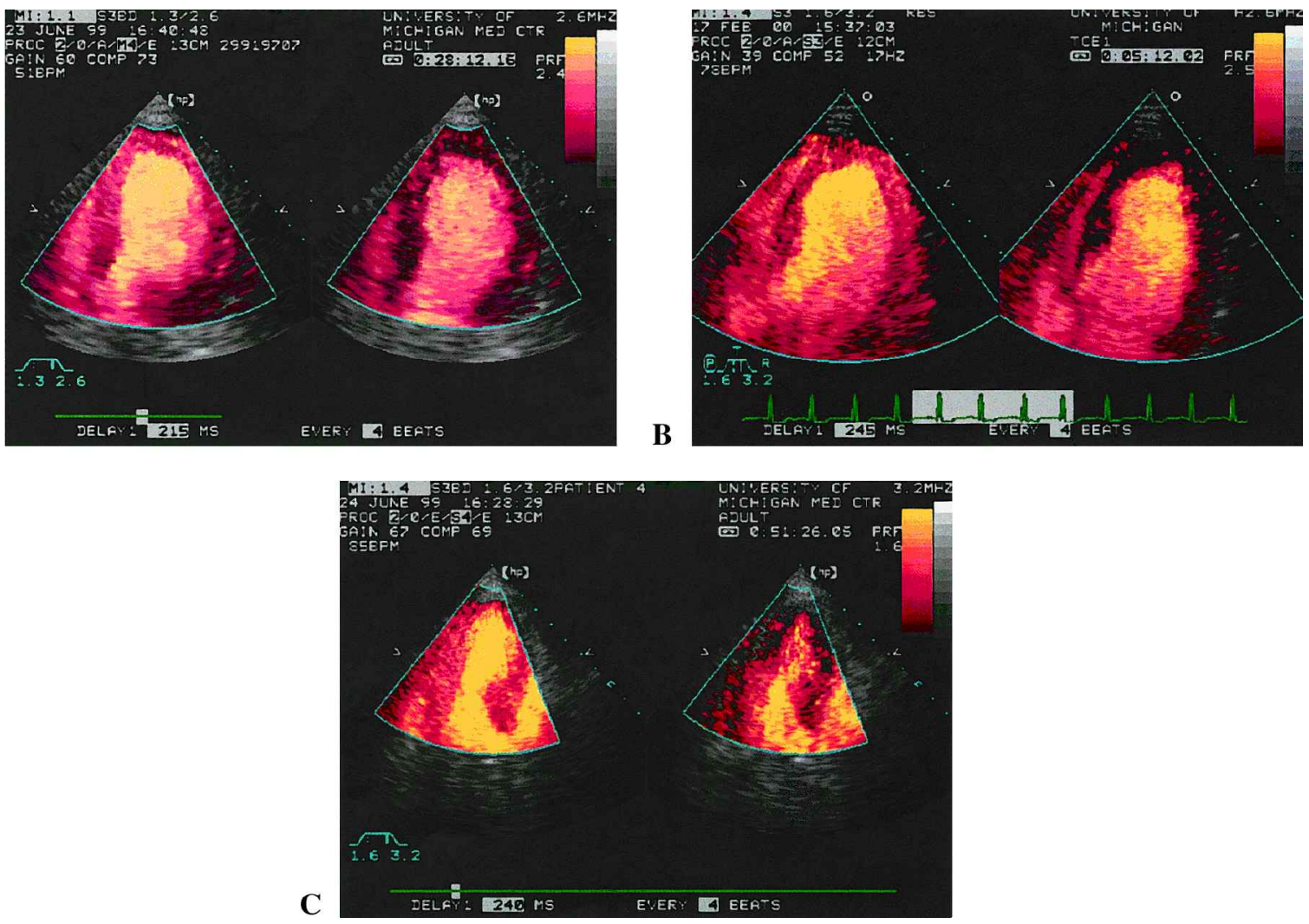

Figure 1. Harmonic Angio MFT using a continuous infusion of Optison is shown in three healthy volunteers in the A. A4C, B. A2C, and C. APLAX views. The left frame in each panel represents the fill frame and the right frame represents the destruction frame. There is readily visible homogenous opacification in the fill frames and markedly reduced opacification in the destruction frames. $A 4 C=$ apical four-chamber; $A 2 C=$ apical two-chamber; $M F T=$ multiple frame triggering.

healthy volunteers also resulted in noticeable myocardial opacification and microbubble destruction. Fill and destruction frames from a representative healthy volunteer are shown in Figure 3, (apical four-chamber view, apical two-chamber view, and apical long-axis view,

A
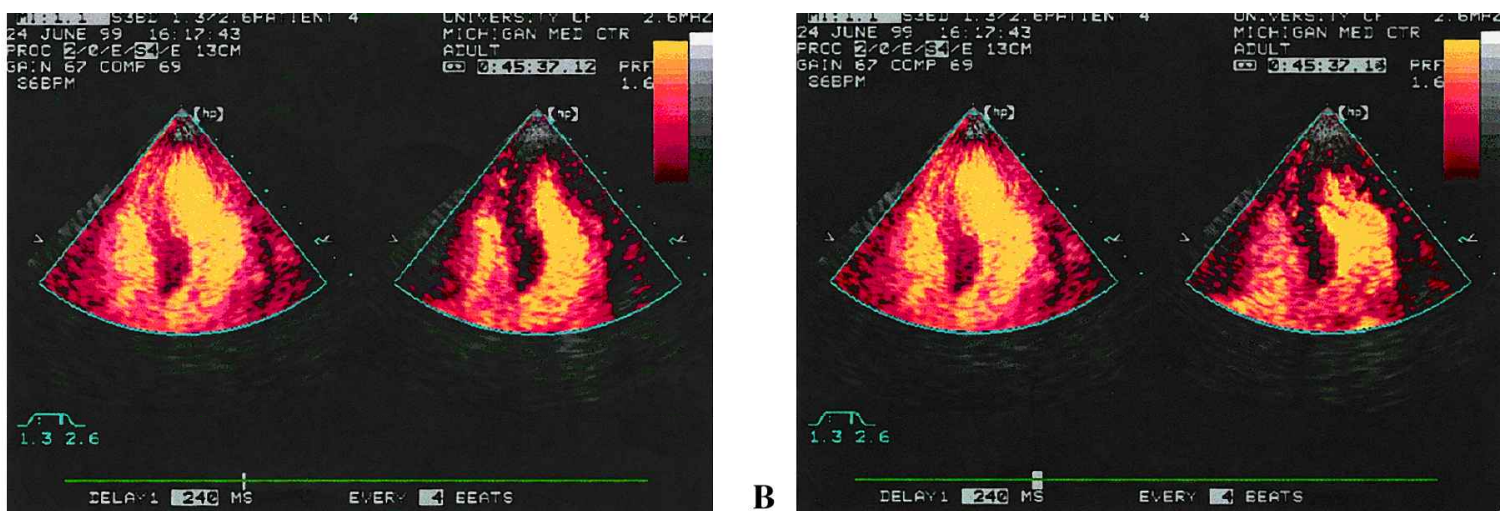

Figure 2. Harmonic Angio MFT in the A4C view using a continuous infusion of Optison is shown in a healthy volunteer. The left frame in each panel is the fill frame and the right is the destruction frame. A. Shows MFT with the destruction frame set at the first available frame after the triggered fill frame, while $\mathbf{B}$. has the destruction frame set at the second available frame after the triggered fill frame. The interval between frames is determined by the continuous imaging frame rate. This showed that there was uniform and near complete microbubble destruction even in the first available frame after the triggered fill frame. $A 4 C=$ apical four-chamber; $M F T=$ multiple frame triggering. 
$\mathbf{A}$
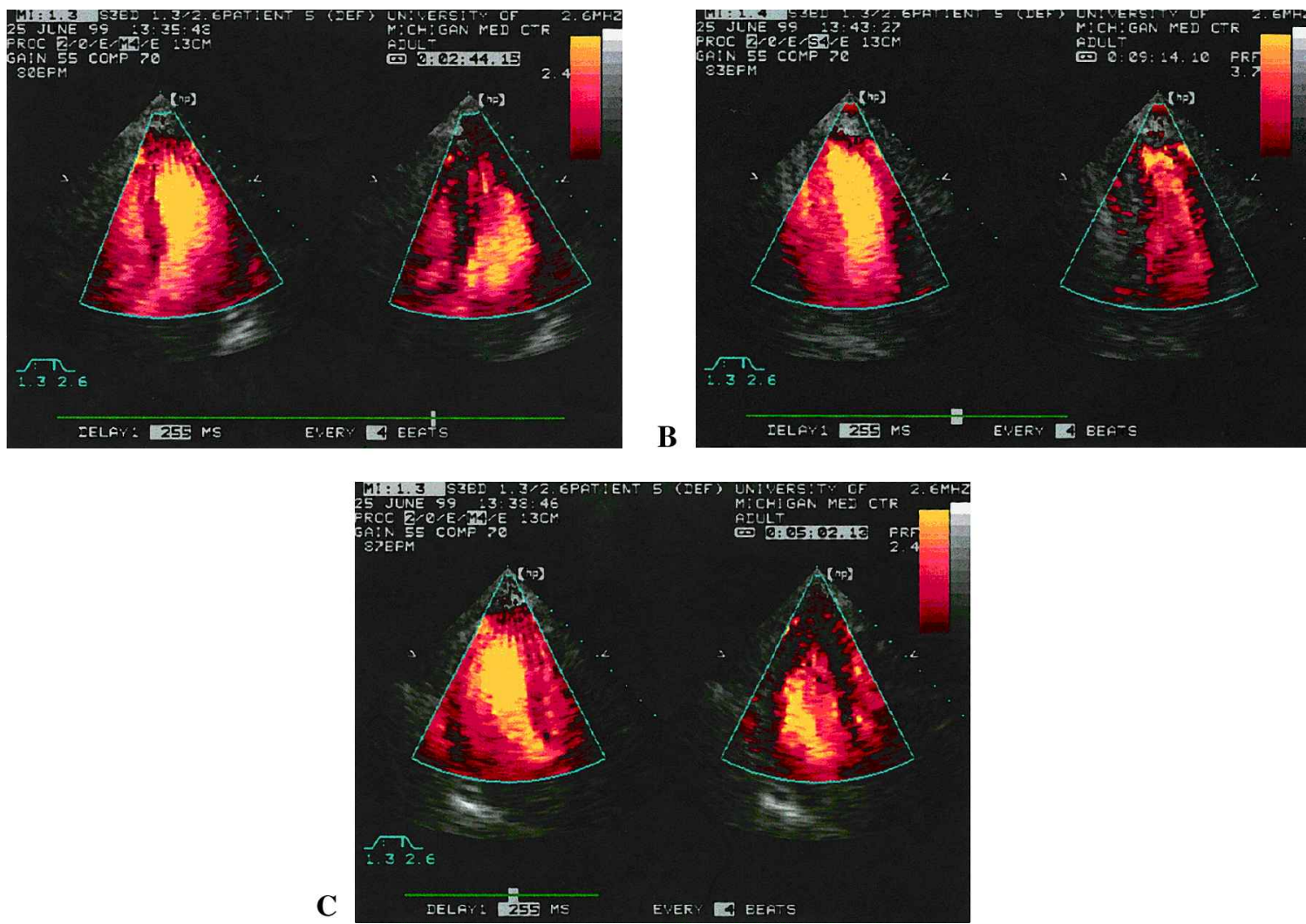

Figure 3. Harmonic Angio MFT in the A. A4C, B. A2C, and C. APLAX views using a continuous infusion of Definity in a healthy volunteer. There is readily visible opacification in the fill frame (left) and markedly reduced visible opacification in the destruction frame (right). A4C = apical four-chamber; A2C = apical two-chamber; APLAX = apical long-axis; $M F T=$ multiple frame triggering.

respectively). There appeared to be near total absence of microbubble signal in the destruction frame, with reasonably uniform opacification in the fill frames.

Harmonic Angio MFT with Optison in the myocardial infarction patients resulted in MCE perfusion defects that corresponded to the defects seen on Sestamibi SPECT imaging, as shown in a representative patient with an apical perfusion defect by SPECT (Fig. 4). The destruction frames in these patients produced uniform and near complete destruction of microbubbles.

\section{Discussion}

This study demonstrated that this new Harmonic Angio MCE technique produced distinct myocardial opacification compared to baseline. In patients with known perfusion defects, decreased myocardial opacification was noted in segments corresponding to SPECT images. Furthermore, this improved MFT algorithm produced destruction frames with uniform and near complete microbubble destruction.

Previous versions of MFT resulted in heterogeneous microbubble destruction due to the nonuniform power in the field. More uniform and complete microbubble destruction was accomplished by the use of an improved version of MFT. This version produced more uniform power in the imaging field by using a lower transmit frequency of $1.3 \mathrm{MHZ}$ in conjunction with an improved transducer.

Harmonic Angio is subject to artifacts in the fill frame from myocardial motion and large blood vessel flow. This study used a more efficient algorithm, which involved reducing the number of pulses per packet from 8 to 5 in conjunction with a more stringent wall filter. Power Doppler techniques are subject to artifacts from myocardial motion between frames. This effect is compounded by the need to image the second or third available frame after the triggered fill frame in order to obtain more complete destruction frames. Since this version 

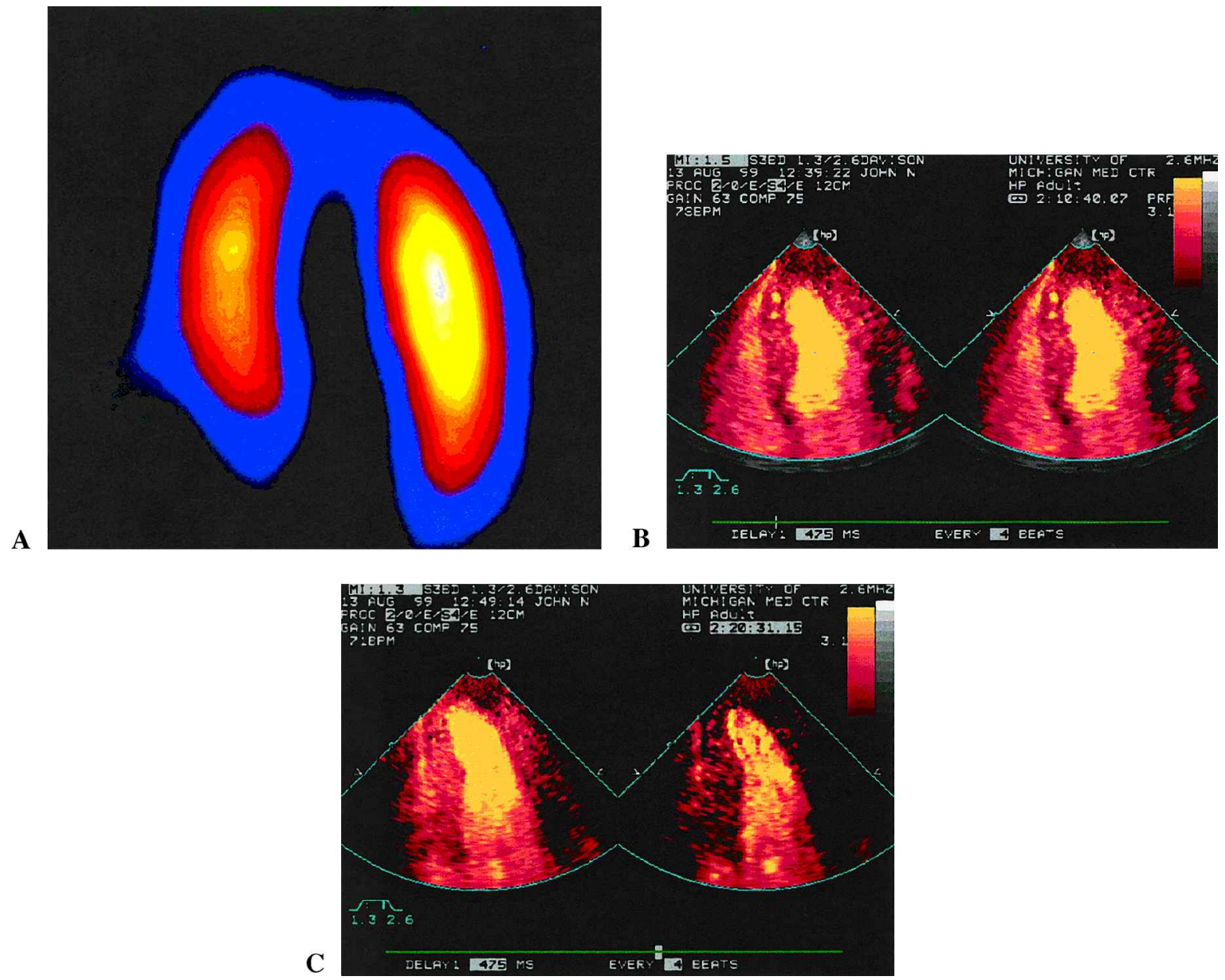

Figure 4. A. Harmonic Angio MFT in a patient with a known apical perfusion defect by SPECT imaging using a continuous infusion of Optison resulted in B. an area of reduced apical opacification with both the A4C (both fill frames) and $\mathbf{C}$. A2C (fill and destruction frames) views. A4C = apical four-chamber; $A 2 C=$ apical two-chamber; $M F T=$ multiple frame triggering.

of Harmonic Angio MFT had more uniform power in the field and was very efficient at destroying microbubbles, the first frame after the fill frame had near total microbubble destruction. Thus, by minimizing the time interval between the fill and destruction frames and by using a smaller number of pulses per packet, this technique minimized myocardial motion between frames, and thus reduced motion artifact.

This study is limited by the use of only one triggering interval (1:4). Higher triggering intervals would have resulted in greater microbubble concentrations in the fill frame and theoretically would have resulted in less complete destruction frames. Also, the use of only one triggering interval does not allow for the assessment of myocardial blood flow. ${ }^{6}$ Although motion and large vessel flow artifacts were minimized in this study, it should be noted that we only performed resting MCE. Stress MCE could produce more motion artifacts due to increased myocardial contractility, and thus, it remains to be seen how efficiently this system will be able to reduce these artifacts. An important limitation of this feasibility study is the lack of comparison between the improved transducer and algorithm to more standard transducers and techniques. Additional limitations include the small number of subjects, the lack of SPECT data in the healthy volunteers, and the exclusion of patients with inadequate echocardiographic windows.

In conclusion, this newer version of Harmonic Angio delineates myocardial perfusion patterns in normal volunteers and patients with a history of myocardial infarction. Furthermore, Harmonic Angio MFT reduces artifacts from cardiac motion and blooming artifacts, resulting in more complete and uniform 
microbubble destruction in the destruction frame. This more efficient version of Harmonic Angio MFT improves the utility of dual triggered, power Doppler-based MCE techniques and may have important implications for clinical MCE trials.

Acknowledgement: We would like to gratefully acknowledge Patrick Rafter, M.S. and Jody Perry, M.Eng. of Agilent Technologies for their role in developing Harmonic Angio MFT and for their expert technical assistance during the myocardial contrast echocardiography studies.

\section{References}

1. Rubin JM, Bude RO, Carson PL, et al: Power Doppler ultrasound: A potentially useful alternative to mean frequency-based color Doppler US. Radiology 1994; 190:853-856.

2. Senior R, Kaul S, Soman P, et al: Power Doppler harmonic imaging: A feasibility study of a new technique for the assessment of myocardial perfusion. Am Heart $J$ 2000;139:245-251.

3. Porter TR, Li S, Jiang L, et al: Real-time visualization of myocardial perfusion and wall thickening in human beings with intravenous ultrasonographic contrast and accelerated intermittent harmonic imaging. J Am Soc Echocardiogr 1999;12:266-271.

4. Murthy TH, Li P, Baisch C, et al: Simulated real-time myocardial contrast echocardiography at a low mechanical index using intravenous Definity ${ }^{\mathrm{TM}}$. (Abstract) J Am Coll of Cardiol 2000;35:460A-461A.

5. Kolias TJ, Avelar E, Lewandowski TJ, et al: Triggered multiple-frame loss of correlation Doppler energy imaging produces nonuniform microbubble destruction: A quantitative videointensity analysis. (Abstract) Eur Heart J 1999;20:33.

6. Wei K, Jayaweera AR, Firoozan S, et al: Quantification of myocardial blood flow with ultrasound-induced destruction of microbubbles administered as a constant venous infusion. Circulation 1998;97:473-483. 